\title{
BIOCHEMICAL ANALYSIS OF FLOWERS OF Vinca major, A MEDICINAL WEED PLANT OF HILLY AREAS OF PAKISTAN
}

Arshad Javaid ${ }^{1 *}$, Malik F. H. Ferdosi ${ }^{2}$, Iqra Haider Khan ${ }^{1}$, Amna Shoaib ${ }^{1}$, Hafiz Muhammad Saeed $^{2}$, Muhammad Abrar Ul Hassan ${ }^{1}$

DOI: https://doi.org/10.28941/pjwsr.v27i4.1014

\begin{abstract}
Vinca major L. is an evergreen perennial weed of family Apocynaceae, growing naturally in hilly areas of Hazara region of Khyber Pakhtunkhwa, Pakistan. Flowers of this weed were collected during June 2021, shade dried and extracted in pure methanol for two weeks. After filtration, the extract was analyzed by GC-MS for the identification of possible bioactive compounds. a-Amyrin was the major compound (32.49\%) followed by lup-20(29)-en-3-ol, acetate, (3ß)- (25.72\%). Moderately abundant compounds included Y-sitosterol $(8.78 \%), \quad \beta$-amyrone $(7.25 \%)$, cyclohexane, 1,3,5-triphenyl- $(7.01 \%)$, olean-12-en-3-ol, acetate, (3ß)- (5.68\%), and $n$-hexadecanoic acid (3.18\%). Some of the identified compounds have various important biological properties including antiinflammatory, antimicrobial, anticancer, antimalarial, antioxidant, antidiabetic, antitumor, etc.
\end{abstract}

Keywords: Bioactive compounds, Flower extract, GC-MS analysis, Khyber Pakhtunkhwa, Vinca major.

Citation: Javaid, A.; M.F.H. Ferdosi; I.H. Khan; A. Shoaib; H.M. Saeed; M.A. Hassan. 2021. Biochemical Analysis of Flowers of Vinca major, A Medicinal Weed Plant of Hilly Areas of Pakistan. Pak. J. Weed Sci. Res., 27(4): 537-546.

\footnotetext{
${ }^{1}$ Department of Plant Pathology, Faculty of Agricultural Sciences, University of the Punjab, Lahore 54590, Pakistan

${ }^{2}$ Department of Horticulture, Faculty of Agricultural Sciences, University of the Punjab, Lahore 54590, Pakistan

*Corresponding author's email: arshad.iags@pu.edu.pk, arshadjpk@yahoo.com
} 


\section{INTRODUCTION}

Indigenous medicinal plants represent a reservoir of natural products that have been used by human beings since ancient times. This has resulted in the use of large number of plants with curative properties against several diseases in many parts of the world (Dar et al., 2017; Jamshidi-Kia et al., 2018; Khan and Javaid, 2020, 2021). Cost and side effects of conventional medications are the major factors responsible for revival of herbal medicines (Uritu et al., 2018). Recent literature also shows the prevalent use of herbs as medicines across the globe (Saravanan et al., 2018; Khan and Javaid, 2019). So far, a lot of important drugs are derived from traditional medicinal herbs (Manandhar et al., 2019; Salmerón-Manzano et al., 2020). A variety of compounds comprised of alkaloids, tannins, steroids, saponins, phenolic acids, terpenoids and quinones have been identified, which possess cardioprotective, anticancer, antioxidant, antimicrobial, anti-insect and a variety of other properties (Tungmunnithum et al., 2018).

Pakistan is enriched in medicinal herbs because of varied climatic conditions (Ullah, 2017; Javed et al., 2021). Many of these medicinal plants have been explored for their antifungal, antibacterial, herbicidal and other bioactive properties (Javaid et al., 2020, 2021a; Banaras et al., 2021; Ferdosi et al., 2021a). These are scattered over a large area and about 600 species known well in Pakistan for their medicinal values (Shinwari, 2010). Vinca major of family Apocynaceae, is an evergreen perennial weed plant that has medicinal and ornamental values (Arora et al., 2010). It grows well in full sun as well as in shade with a height of up to $25 \mathrm{~cm}$ while spreading indefinitely.
Phytochemical screening of $V$. major revealed the presence of saponins, alkaloids, phenols, organic acids and sterols exhibiting antioxidant, antimicrobial, antidiabetic and hypotensive properties (Singh et al., 2014; Wei and Liu, 2021). Vinca extracts have diverse compounds that act as antineoplastic agents and are also used to treat choriocarcinoma, lymphomas and hodgkin's diseases (Vishwakarma and Prajapati, 2019; González-Burgos and Gómez-Serranillos, 2021). Moreover, aerial plant parts are used traditionally to treat diarrhea, piles, leukemia, malaria, diabetes, sore throat, cough and diabetes (Rajput et al., 2011; Ajaib et al., 2014; Comfort et al., 2019). However, studies regarding phytochemical analysis of flowers of this plant from Pakistan are scarce. Thus, the present study was conducted to identify phytoconstituents of $V$. major flowers through GC-MS analysis and to document its medicinal importance.

\section{MATERIALS AND METHODS}

Disease free and mature flowers of $V$. major were collected from Murree, Pakistan. Plucked flowers were packed in plastic bags and shifted to laboratory for further analysis. Flowers were washed in water and completely dried at $35^{\circ} \mathrm{C}$ in a hot air oven. After evaporating moisture from flower, the dried flowers were then finely crushed into a powdered form with pestle and mortar. Ten grams of this material were soaked in $50 \mathrm{~mL}$ of analytical graded methanol and kept for two weeks so that maximum bioactive compounds can be extracted. Thereafter, the extract was filtered through a filter paper. Following filtration, $2 \mathrm{~mm}$ extract was collected in a $5 \mathrm{~mL}$ vial for GC-MS analysis. 


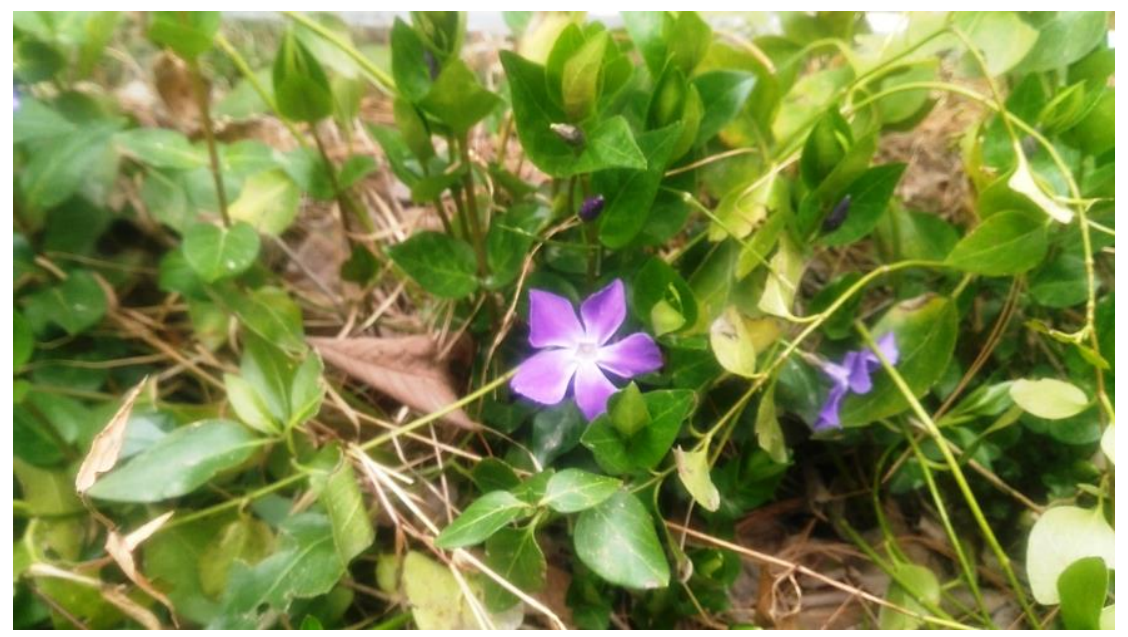

Fig. 1: Vinca major growing in Murree, Pakistan.

Various biologically active
compounds were identified from methanolic flower extract using GC-MS analysis as described by Ferdosi et al. (2020). Gas chromatography (GC) was performed on 7890B Model Machine, Agilent Technologies (USA) with Column DB-5ms having dimensions of $30 \mathrm{~m} \times$ $0.25 \mu \mathrm{m} \times 0.25 \mu \mathrm{m}$. Helium, an inert gas (having 4 amu atomic mass) was used as a carrier gas. Injection volume was $1 \mu \mathrm{L}$, while oven initial temperature was $80^{\circ} \mathrm{C}$ that was raised to $10^{\circ} \mathrm{C} \mathrm{min}^{-1}$ up to $300{ }^{\circ} \mathrm{C}$. MS analysis was performed on 5977A Model Machine, Agilent Technologies (USA) with scan range of $50-500 \mathrm{~m} / \mathrm{z}$; solvent delay time was 5 min. Source temperature was 230 ${ }^{\circ} \mathrm{C}$ with $50 \mathrm{~min}$ run time. The resulted spectrum was then analyzed with NIST library of 2017 version for the phytochemical characterization and the compounds were arranged in the ascending order of their retention times. Relative abundance of the compounds was analyzed using of peaks heights in the chromatogram. Chemical structures of various compounds in the extract were drawn using ChemDraw software.

In order to collect information on biological activities of the identified compounds, different databases including Science Direct, PubMed, SciELO, Google Scholar, Directory of Open Access Journals and Crossref were surveyed.

\section{RESULTS AND DISCUSSION}

Fourteen compounds were
detected in GC-MS analysis of methanolic flower extract of $V$. major (Fig. 2). Details of the identified compounds are presented in Table 1 . The most abundant compound was aamyrin $(32.49 \%)$ followed by lup20(29)-en-3-ol, acetate, (3ß)$(25.72 \%)$. Four compounds namely $\mathrm{Y}$ sitosterol $(8.78 \%), \beta$-amyrone $(7.25 \%)$, cyclohexane, 1,3,5-triphenyl- (7.01\%), olean-12-en-3-ol, acetate, (3ß)$(5.68 \%)$ and $n$-hexadecanoic acid $(3.18 \%)$ were ranked as moderately abundant compounds. Compounds such as campesterol $(2.11 \%)$, cyclododecane $(1.17 \%)$, pentadecanoic acid, 14methyl-, methyl ester (1.32\%), heneicosane $(1.31 \%)$, 2-ethylacridine $(1.23 \%), 1,2$-bis(trimethylsilyl)benzene $(1.42 \%)$, were categorized as less abundant ones. Structures of major compounds are shown in Fig. 3.

The principal compound in the flower extract was a-amyrin. Earlier, this compound has been reported in Cirsium arvense (Ferdosi et al., 2021b), Myrcianthes pungens (Cardoso et al., 2020) and Strobilanthes callosus (Singh et al., 2002). It is known for its antimicrobial, antioxidant and antiinflammatory properties (Singh et al., 2002). In addition, it also showed inhibitory effects against human oxidosqualene cyclase (Chen et al., 2017). Its pharmacological activity is also known in gastrointestinal tract and immunological system (Nogueira et al., 2019), and in the treatment of gingivitis and periodontitis (Pinto et al., 2008). Likewise, $\beta$-amyrone isolated from oil- 
resins of Protium paniculatum also showed anti-inflammatory activity (de Almeida et al., 2015).

The second most abundant compound lup-20(29)-en-3-ol, acetate, $(3 \beta)-$, also known as lupeyl acetate and lupeol acetate, belongs to triterpenoids group. This compound was isolated from bark of Artocarpus integra and showed anticancer activity against breast cancer cells MCF-7 (Suwito et al., 2016). It also possesses numerous other biological activities including anti-inflammatory, antituberculosis, antimalarial, antimicrobial and antinociceptive (Prachayasittikul et al., 2010; Chen et al., 2012), An isomer of this compound, olean-12-en-3-ol, acetate, (3ß)- also known as $\beta$-amyrin 3- acetate, was found as a moderately occurring compound in this study. It possesses anti-inflammatory potential and also inhibits growth of Staphylococcus aureus (Hichri et al., 2003; Akihisa et al., 2010). It has been found as a major constituent in flowers of $C$. arvense and (Fernandes et al., 2013; Ferdosi et al., 2021b).

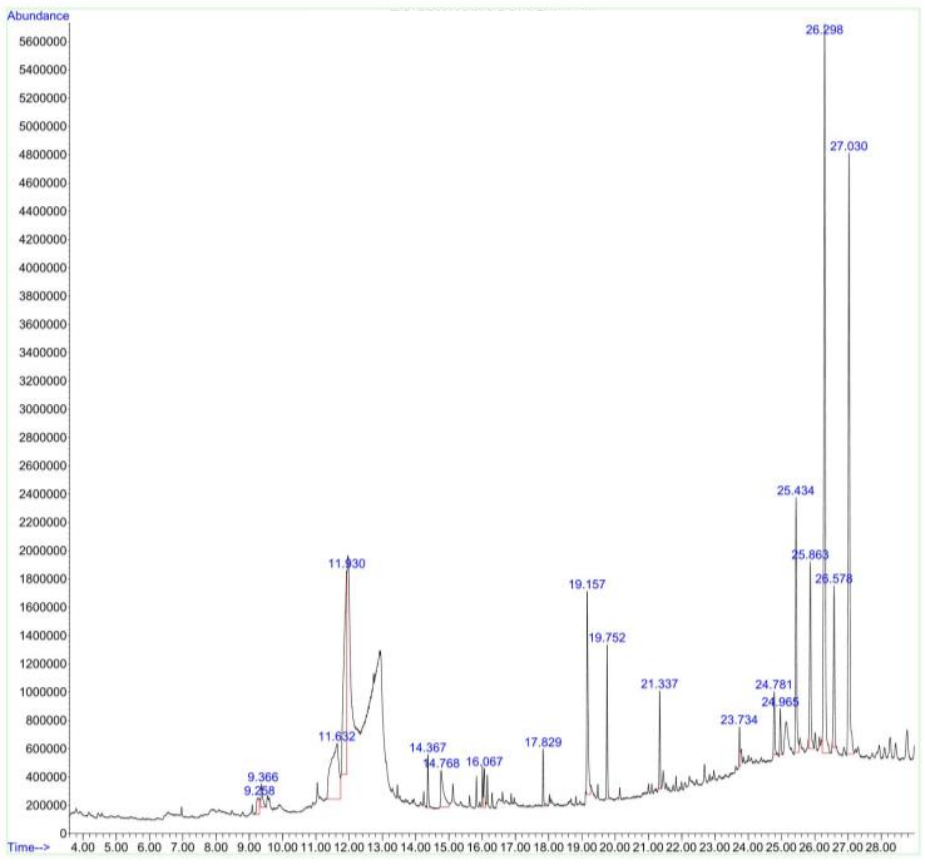

Fig. 1: GC-MS chromatogram of methanolic flower extract of Vinca major.

Table 1: Compounds identified in methanolic flower extract of Vinca major through GCMS analysis.

\begin{tabular}{|c|c|c|c|c|c|}
\hline $\begin{array}{l}\text { Sr. } \\
\text { No. }\end{array}$ & Names of compounds & $\begin{array}{l}\text { Molecular } \\
\text { formula }\end{array}$ & $\begin{array}{l}\text { Molecular } \\
\text { weight }\end{array}$ & $\begin{array}{l}\text { Retention } \\
\text { time (min) }\end{array}$ & $\begin{array}{c}\text { Peak } \\
\text { area } \\
(\%)\end{array}$ \\
\hline 1 & 5-Dodecene, (E)- & $\mathrm{C}_{12} \mathrm{H}_{24}$ & 168.31 & 9.366 & 1.17 \\
\hline 2 & $\begin{array}{l}\text { Pentadecanoic acid, 14- } \\
\text { methyl-, methyl ester }\end{array}$ & $\mathrm{C}_{17} \mathrm{H}_{34} \mathrm{O}_{2}$ & 270.45 & 14.367 & 1.32 \\
\hline 3 & n-Hexadecanoic acid & $\mathrm{C}_{16} \mathrm{H}_{32} \mathrm{O}_{2}$ & 256.42 & 14.768 & 3.18 \\
\hline 4 & $\begin{array}{l}9,12,15 \text {-Octadecatrienoic } \\
\text { acid, methyl ester, }(Z, Z, Z) \text { - }\end{array}$ & $\mathrm{C}_{19} \mathrm{H}_{32} \mathrm{O}_{2}$ & 292.45 & 16.067 & 1.25 \\
\hline 5 & Heneicosane & $\mathrm{C}_{21} \mathrm{H}_{44}$ & 296.57 & 17.829 & 1.31 \\
\hline 6 & $\begin{array}{l}\text { Cyclohexane, 1,3,5- } \\
\text { triphenyl- }\end{array}$ & $\mathrm{C}_{24} \mathrm{H}_{24}$ & 312.44 & 19.157 & 7.01 \\
\hline 7 & 2-Ethylacridine & $\mathrm{C}_{15} \mathrm{H}_{13} \mathrm{~N}$ & 207.27 & 23.734 & 1.23 \\
\hline 8 & Campesterol & $\mathrm{C}_{28} \mathrm{H}_{48} \mathrm{O}$ & 400.68 & 24.781 & 2.11 \\
\hline 9 & 1,2-Bis(trimethylsilyl) & $\mathrm{C}_{12} \mathrm{H}_{22} \mathrm{Si}_{2}$ & 222.47 & 24.965 & 1.42 \\
\hline
\end{tabular}


benzene

$10 \quad \mathrm{Y}$-Sitosterol

$11 \beta$-Amyrone

$\mathrm{C}_{29} \mathrm{H}_{50} \mathrm{O}$

414.70

25.434

8.78

12 a-Amyrin

$\mathrm{C}_{30} \mathrm{H}_{48} \mathrm{O}$

424.70

25.863

7.25

13 Olean-12-en-3-ol, acetate,

$\mathrm{C}_{30} \mathrm{H}_{50} \mathrm{O}$

426.71

26.298

32.49 (3ß)-

14 Lup-20(29)-en-3-ol,

$\mathrm{C}_{32} \mathrm{H}_{52} \mathrm{O}_{2} \quad 468.75$

26.578

5.68

\section{acetate, $(3 \beta)$ -}

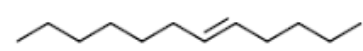

5-Dodecene, (E)-<smiles>COC(=O)CCCCCCCCCCCC(C)C</smiles>

Pentadecanoic acid, 14methyl-, methyl ester<smiles>CC/C=C/C=C/C=C/CCCCCCC(=O)OC</smiles>

9,12,15-Octadecatrienoic acid, methyl ester, $(Z, Z, Z)-$<smiles>CCc1ccc2nc3ccccc3cc2c1</smiles>

2-Ethylacridine

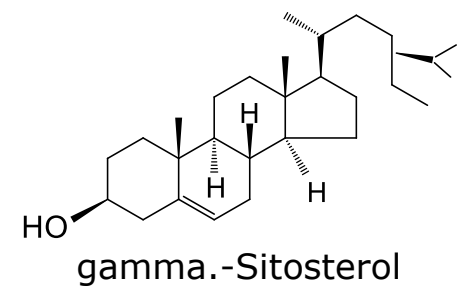

gamma.-Sitosterol

\section{Heneicosane}

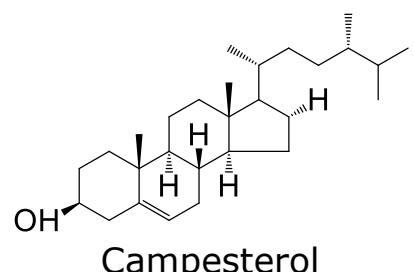

Campesterol

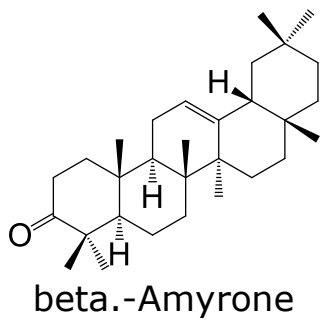

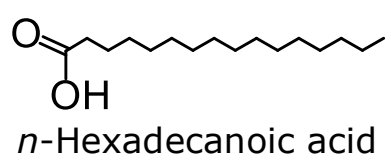

n-Hexadecanoic acid<smiles>c1ccc(C2CC(c3ccccc3)CC(c3ccccc3)C2)cc1</smiles>

Cyclohexane, 1,3,5triphenyl-<smiles>Cc1ccccc1C(C)C</smiles>

1,2-Bis(trimethylsilyl) benzene

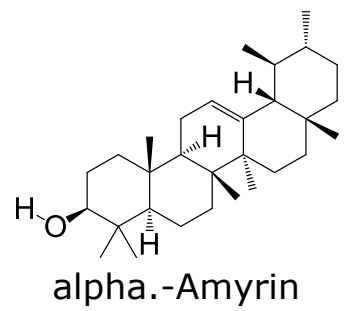

Olean-12-en-3-ol, acetate, (3.beta.)-

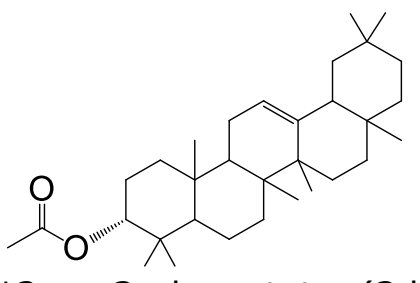

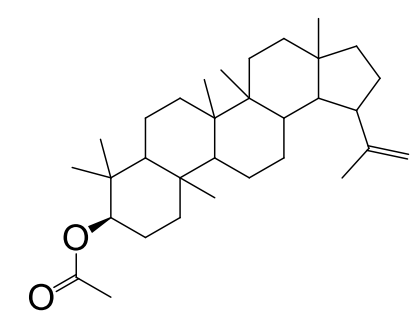

Lup-20(29)-en-3-ol, acetate, (3.beta.)-

Fig. 3: Structures of compounds present in flower extract of Vinca major.

Plant species such as Lippia nodiflora and Acacia nilotica, with anticancer (Sundarraj et al., 2012) and antidiabetic properties (Balamurugan et al., 2011). 9,12,15-Octadecatrienoic acid, methyl ester, $(Z, Z, Z)-$ and pentadecanoic acid, 14-methyl-, methyl ester are fatty acid methyl esters, which have been found in numerous plant species such as Cannabis sativa, 
Ageratum conyzoides and Coronopus didymus (Banaras et al., 2021; Javaid et al., 2018, 2021b). Such compounds showed antifungal and antibacterial activities (Johnson et al., 2011; Bashir et al., 2012). n-Hexadecanoic acid, also known as palmitic acid, is a highly biologically active compound, found in many plants (Javaid et al., 2018; Naqvi et al., 2020). It possesses antiinflammatory, antimicrobial, mosquito larvicidal, hypocholesterolemic, antioxidant and pesticidal properties (Rahuman et al., 2000; Kumar et al.,
2010; Aparna et al., 2012; Abubakar and Majinda, 2016). Heneicosane, an alkane, isolated from Plumbago zeylanica showed antimicrobial activity against Streptococcus pneumoniae and Aspergillus fumigatus (Vanitha et al., 2020). Likewise, 2-ethylacridine also possesses antimicrobial and antitumor activities (Vijayakumari and Raj, 2019). Campesterol is a sterol found in plant and cholesterol lowering and anticarcinogenic properties (Choi et al., 2007).

Table 2: Bioactivity of components of methanolic flower extract of Vinca major.

\begin{tabular}{|c|c|c|c|}
\hline Sr. No. & Names of compounds & Bioactivity & Reference \\
\hline 1 & 5-Dodecene, (E)- & - & - \\
\hline 2 & $\begin{array}{l}\text { Pentadecanoic acid, 14- } \\
\text { methyl-, methyl ester }\end{array}$ & $\begin{array}{l}\text { Antifungal, } \\
\text { Antimicrobial }\end{array}$ & Bashir et al. (2012) \\
\hline 3 & n-Hexadecanoic acid & $\begin{array}{c}\text { Antioxidant, } \\
\text { pesticidal, anti- } \\
\text { inflammatory, } \\
\text { mosquito larvicide, } \\
\text { hemolytic }\end{array}$ & $\begin{array}{l}\text { Kumar et al. (2010); } \\
\text { Aparna et al. (2012); } \\
\text { Abubakar and } \\
\text { Majinda (2016) }\end{array}$ \\
\hline 4 & $\begin{array}{l}9,12,15 \text {-Octadecatrienoic } \\
\text { acid, methyl ester, }(Z, Z, Z) \text { - }\end{array}$ & $\begin{array}{c}\text { Antibacterial, } \\
\text { antioxidant, } \\
\text { anticancer, } \\
\text { antipyretic, } \\
\text { cardioprotective, } \\
\text { antiarthritic neural } \\
\text { function, } \\
\text { Antiandrogenic, }\end{array}$ & $\begin{array}{l}\text { Johnson et al. } \\
\text { (2011); Akpuaka et } \\
\text { al. (2013) }\end{array}$ \\
\hline 5 & Heneicosane & Antimicrobial & Vanitha et al. (2020) \\
\hline 6 & $\begin{array}{l}\text { Cyclohexane, 1,3,5- } \\
\text { triphenyl- }\end{array}$ & - & - \\
\hline 7 & 2-Ethylacridine & $\begin{array}{l}\text { Antimicrobial, } \\
\text { antitumor }\end{array}$ & $\begin{array}{l}\text { Vijayakumari and Raj } \\
\text { (2019) }\end{array}$ \\
\hline 8 & Campesterol & $\begin{array}{l}\text { Anti-cholesterol, } \\
\text { anticarcinogenic }\end{array}$ & Choi et al. (2007) \\
\hline 9 & $\begin{array}{l}1,2 \text {-Bis(trimethylsilyl) } \\
\text { benzene }\end{array}$ & - & - \\
\hline 10 & Y-Sitosterol & $\begin{array}{l}\text { Anticancer, } \\
\text { antidiabetic }\end{array}$ & $\begin{array}{l}\text { Balamurugan et al. } \\
\text { (2011); Sundarraj et } \\
\text { al. (2012) }\end{array}$ \\
\hline 11 & $\beta$-Amyrone & Anti-inflammatory & $\begin{array}{l}\text { de Almeida et al. } \\
(2015)\end{array}$ \\
\hline 12 & a-Amyrin & $\begin{array}{c}\text { Antimicrobial } \\
\text { Anti-inflammatory, } \\
\text { antioxidant, inhibitor } \\
\text { of human } \\
\text { oxidosqualene } \\
\text { cyclase }\end{array}$ & $\begin{array}{l}\text { Singh et al. (2002); } \\
\text { Chen et al. (2017); } \\
\text { Cardoso et al. (2020) }\end{array}$ \\
\hline 13 & $\begin{array}{l}\text { Olean-12-en-3-ol, acetate, } \\
(3 \beta)-\end{array}$ & $\begin{array}{l}\text { Antibacterial, anti- } \\
\text { inflammatory }\end{array}$ & $\begin{array}{l}\text { Hichri et al. (2003); } \\
\text { Akihisa et al., (2010) }\end{array}$ \\
\hline 14 & Lup-20(29)-en-3-ol, & Anticancer, anti- & Prachayasittikul et al. \\
\hline
\end{tabular}


acetate, $(3 \beta)$ -

inflammatory, (2010); Chen et al. antituberculosis, antimalarial, antimicrobial, antinociceptive (2012); Suwito et al. (2016)

\section{Conclusion}

Flower of $V$. major is a rich source of bioactive substances especially $n$ hexadecanoic acid; a-amyrin, lupeol acetate, $\mathrm{Y}$-sitosterol; heneicosane and

fatty acid methyl esters with a number of biological activities including anticancer and antidiabetic. 


\section{REFERENCES CITED}

Abubakar, M.N. and R.R.T. Majinda. 2016. GC-MS Analysis and preliminary antimicrobial activity of Albizia adianthifolia Schumach and Pterocarpus angolensis. Medicines, 3: Article 3.

Ajaib, M., S.K. Haider, A. Zikrea and M.F. Siddiqui. 2014. Ethnobotanical studies of herbs of Agra valley Parachinar, Upper Kurram Agency, Pakistan. Int. J. Biol. Biotechnol., 11: 71-83.

Akihisa, T., N. Kojima, T. Kikuchi, K. Yasukawa, H. Tokuda, E. Masters, A. Manosroi and J. Manosroi. 2010. Anti-inflammatory and chemopreventive effects of triterpene cinnamates and acetates from shea fat. J. Oleo Sci., 59: 273-280.

Akpuaka, A., M.M Ekwenchi, D.A. Dashak and A. Dildar. 2013. Biological activities of characterized isolates of $n$-hexane extract of Azadirachta indica A. Juss (Neem) leaves. Nat. Sci., 11(5):142-145.

Aparna, V., D. Vijayan, P. Mandal and P. Karthe. 2012. Anti-inflammatory property of $n$-hexadecanoic acid: Structural evidence and kinetic assessment. Chem. Biol. Drug Des., 80: 434-439.

Arora, R., A.K. Mathur, A. Mathur and C.M. Govil. 2010. Biotechnology of Himalayan Vinca major and $V$. minor. In: Medicinal Plant Biotechnology, CABI Publishers UK. pp. 207-221.

Balamurugan, R., V. Duraipandiyan and S. Ignacimuthu. 2011. Antidiabetic activity of $\mathrm{Y}$-sitosterol isolated from Lippia nodiflora L. in streptozotocin induced diabetic rats. Eur. J. Pharmacol., 667: 410-418.

Banaras, S., A. Javaid and I.H. Khan. 2021. Bioassays guided fractionation of Ageratum conyzoides extract for the identification of natural antifungal compounds against Macrophomina phaseolina. Int. J. Agric. Biol., 25(4): 761-767.

Bashir, A., K. Ibrar, B. Shumaila and S. Azam. 2012. Chemical composition and antifungal, phytotoxic, brine shrimp cytotoxicity, insecticidal, and antibacterial activities of the essential oils of Acacia modesta. J. Med. Plants Res., 6: 4653-4659.

Cardoso, B.K., H.L.M. de Oliveira, U.Z. Melo, C.M.M. Fermandez, C.F.A.A. Campo and J.E. Goncalves. 2020. Antioxidant activity of $a-$ and $\beta$ amyrin isolated from Myrcianthes pungens leaves. Nat. Prod. Res., 34: 1777- 1781.

Chen, Y.F., C. Ching, T.S. Wu, C.R. Wu, W.T. Hsieh and H.Y. Tsai. 2012. Balanophora spicata and lupeol acetate possess antinociceptive and anti-inflammatory activities in vivo and in vitro. Evid. Based Complement. Altern. Med., 2012: Article 371273.

Chen, D., F. Xu, P. Zhang, J. Deng, H. Sun, X. Wen and J. Liu. 2017. Practical synthesis of a-amyrin, $\beta$ amyrin, and lupeol: The potential natural inhibitors of human oxidosqualene cyclase. Arch. Pharm. (Weinheim), 350: doi: 10.1002/ardp.201700178.

Choi, J-M., E-O. Lee, H-J. Lee and S-H. Kim. 2007. Identification of campesterol from Chrysanthemum coronarium L. and its antiangiogenic activities. Phytother. Res., 21: 954-959.

Comfort, M.I., D. Majesty, A. Eze, N. Kelechi, E. Ahamefula, N. Ijeoma and O. Emmanuel. 2019. Effect of ethanolic leaf extract of Vinca major L. on biochemical parameters and glucose level of alloxan induced diabetic rats. Afr. J. Biotechnol., 18: 1054-1068.

Dar, R.A., M. Shahnawaz and P.H. Qazi. 2017. General overview of medicinal plants: A review. J. Phytopharmacol., 6: 349-351.

de Almeida, P.D.O., A.P.A. Boleti, A.L Rüdiger, G.A. Lourenço, V.F. da V. Junior and E.S. Lima. 2015. Antiinflammatory activity of triterpenes isolated from Protium paniculatum oil-resins. Evid. Based Complement. Altern. Med., 2015: Article 293768.

Ferdosi, M.F.H., I.H. Khan, A. Javaid, T. Sattar and A. Munir. 2020. Identification of antimicrobial constituents in essential oil of 
Paulownia fortunei flowers. Mycopath, 18(2): 53-57.

Ferdosi, M.F.H., I.H. Khan, A. Javaid, M. Nadeem and A Munir. 2021a. Biochemical profile of Calotropis procera flowers. Pak. J. Weed Sci. Res. 27(3): 341-349.

Ferdosi, M.F.H., I.H. Khan, A. Javaid and M.F.A. Fardosi. 2021b. GC-MS examination of methanolic extract of Cirsium arvense flower. Pak. J. Weed Sci. Res., 27(2): 173-180.

Fernandes, C.P., A.L. Correa, J.F.R. Lob, O.P. Caramel, F.B. de Almeida and L. Rocha. 2013. Triterpene esters and biological activities from edible fruits of Manilkara subsericea (Mart.) Dubard, Sapotaceae. Biomed Res. Int., 2013: Article 280810.

González-Burgos, E. and M.P. GómezSerranillos. 2021. Vinca alkaloids as chemotherapeutic agents against breast cancer. Discovery and development of anti-breast cancer agents from natural products. In: Natural Product Drug Discovery, G. Brahmachari (Ed.), Elsevier. pp. 69-101.

Hichri, F., H.B. Jannet, J. Cheriaa, S. Jegham and Z. Mighri. 2003. Antibacterial activities of a few prepared derivatives of oleanolic acid and of other natural triterpenic compounds. C. R. Chim., 6(4):473483.

Jamshidi-Kia, F., Z. Lorigooini and $\mathrm{H}$. Amini-Khoei. 2018. Medicinal plants: Past history and future perspective. J. Herbmed. Pharmacol., 7: 1-7.

Javaid, A., U. Latif, N. Akhtar, D. Ahmed and S. Perveen. 2018. Molecular characterization of Fusarium moniliforme and its management by methanolic extract of Coronopus didymus. Pak. J. Bot. 50(5): 20692075.

Javaid, N., M.H. Shah, I.H. Khan, A. Javaid and S.M. Waleed. 2020. Herbicidal activity of Ageratum conyzoides against parthenium. Pak. J. Weed Sci. Res., 26(2):137146.

Javaid, A., S.F. Naqvi and I.H. Khan. 2021a. Ethyl acetate extract of Chenopodium murale root, a source of bioactive compounds. Pak. J. Weed Sci. Res., 27(1): 93100.

Javaid, A., I.H. Khan and M.F.H. Ferdosi. 2021b. Bioactive constituents of wild Cannabis sativa roots from Pakistan. Pak. J. Weed Sci. Res., 27(3): 359-368.

Javed, S., Z. Mahmood, K.M. Khan, S.D. Sarker, A. Javaid, I.H. Khan and A. Shoaib (2021). Lupeol acetate as a potent antifungal compound against opportunistic human and phytopathogenic mold Macrophomina phaseolina. Sci. Rep., 11: 8417.

Johnson, M., Y. Mariswamy and W.F. Gnaraj. 2011. Chromatographic finger print analysis of steroids in Aerva lanasa L. by HPTLC technique. Asian Pal. J. Trop. Biomed., 1: 428-433.

Khan, I.H. and A. Javaid. 2019. Antifungal, antibacterial and antioxidant components of ethyl acetate extract of quinoa stem. Plant Prot., 3(3): 125-130.

Khan, I.H. and A. Javaid. 2020. Anticancer, antimicrobial and antioxidant compounds of quinoa inflorescence. Adv. Life Sci., 8(1): 68-72.

Khan, I.H. and A. Javaid. 2021. Identification of biologically important compounds in neem leaves through GC-MS analysis. Jordan J. Pharm. Sci., 14(3): 359366.

Kumar, P.P., S. Kumaravel and C. Lalitha. 2010. Screening of antioxidant activity, total phenolics and GC-MS study of Vitex negundo. Afr. J. Biochem. Res., 4: 191-195.

Manandhar, S., S. Luitel and R.K. Dahal. 2019. In vitro antimicrobial activity of some medicinal plants against human pathogenic bacteria. J. Trop. Med., 2019: Article 1895340.

Naqvi, S.F., I.H. Khan and A. Javaid. 2020. Hexane soluble bioactive components of Chenopodium murale stem. Pak. J. Weed Sci. Res., 26(4): 425-432.

Nogueira, A.O., Y.I.S. Oliveira, B.L. Adjafre, M.E.A de Moraes and G.F. Aragao. 2019. Pharmacological effects of the isomeric mixture of 
alpha and beta amyrin from Protium heptaphyllum: a literature review. Fundam. Clin. Pharmacol., 33: 4-12.

Pinto H.S.A., L.M.S. Pinto, G.M.A. Cunha, M.H. Chaves, F.A. Santos and V.S. Rao. 2019. Antiinflammatory effect of $a, \beta$-amyrin, a pentacyclic triterpene from Protium heptaphyllum in rat model of acute periodontitis. Inflammopharmacology, 16: 4852.

Prachayasittikul, S., P. Saraban, R. Cherdtrakulkiat, S. Ruchirawat and V. Prachayasittikul. 2010. New bioactive triterpenoids and antimalarial activity of Diospyros rubra Lec. EXCLI J., 9: 1-10.

Rahuman, A.A., G. Gopalakrishnan, B.S. Ghouse, S. Arumugam and $B$. Himalayan. 2000. Effect of Feronia limonia on mosquito larvae. Fitoterapia, 71: 553-555.

Rajput, M.S., V. Nair, A. Chauhan, H. Jawanjal and V. Dange. 2011. Evaluation of antidiarrheal activity of aerial parts of Vinca major in experimental animals. Middle-East J. Sci. Res., 7: 784-788.

Salmerón-Manzano, E., J.A. GarridoCardenas and F. ManzanoAgugliaro. 2020. Worldwide research trends on medicinal plants. Int. J. Environ. Res. Public Health, 17: Article ID 3376.

Saravanan, M., P. Senthilkumar, K. Kalimuthu, V. Chinnadurai, S. Vasantharaj and A. Pugazhendhi. 2018. Phytochemical and pharmacological profiling of Turnera subulata Sm., a vital medicinal herb. Ind. Crop. Prod., 124: 822-833.

Shinwari, Z.K. 2010. Medicinal plants research in Pakistan. J. Med. Plant Res., 4: 161-176.

Singh, B., P.M. Sahu and M.K. Sharma. 2002. Anti-inflammatory and antimicrobial activities of triterpenoids from Strobilanthes callosus Nees. Phytomedicine, 9: 355-359.

Singh, S., J. Gupta and S.S. Kanwar. 2014. Antilipase, antiproliferatic and antiradical activities of methanolic extracts of Vinca major. J. Pharm. Phytochem., 3: 53-64.

Sundarraj, S., R. Thangam, V. Sreevani, K. Kaveri, P. Gunasekaran, S. Achiraman and S. Kannan. 2012. $Y$-Sitosterol from Acacia nilotica $L$. induces G2/M cell cycle arrest and apoptosis through c-Myc suppression in MCF-7 and A549 cells. J. Ethnopharmacol., 141: 803-809.

Suwito, H., W.L. Heffen, H. Cahyana, and W.P. Suwarso. 2016. Isolatio $\mathrm{n}$, transformation, anticancer, and apoptosis activity of lupeyl acetate from Artocarpus integra. AIP Conf. Proc., 1718: 080004.

Tungmunnithum, D., A. Thongboonyou, A. Pholboon and A. Yangsabai. 2018. Flavonoids and other phenolic compounds from medicinal plants for pharmaceutical and medical aspects: An overview. Medicines, 5: Article ID 93.

Ullah, N. 2017. Medicinal Plants of Pakistan: Challenges and Opportunities. Int. J. Complement. Alternat. Med., 6: Article 00193.

Uritu, C.M., C.T. Mihai, G.D. Stanciu, G. Dodi, T. Alexa-Stratulat, A. Luca and B.I. Tamba. 2018. Medicinal plants of the family Lamiaceae in pain therapy: A review. Pain Res. Manag., 2018: Article ID 7801543.

Vanitha, V., S. Vijayakumar, M. Nilavukkarasi, V.N. Punitha, E. Vidhya and P.K. Praseetha. 2020. Heneicosane-A novel microbicidal bioactive alkane identified from Plumbago zeylanica L. Ind. Crops Prod., 154: 112748.

Vijayakumari, J. and T.L.S. Raj. 2019. GC- MS analysis of secondary metabolites from acetone and chloroform extract of Dicranopteris linearis (Burm. F.) Underw. Int. Res. J. Biol. Sci., 8: 39-43.

Vishwakarma, R. and V. Prajapati. 2019. Drug of Vinca: Used as a anticancer agent". J. Pharmacol. Toxicol., 1: 40-44.

Wei, Q. and R. Liu. 2021. Constituents of essential oil from Vinca major var. variegata and its antibacterial activity. Chem. Nat. Compd., 57: 965-967. 